\title{
De quoi l'agriculture écologiquement intensive est-elle le nom ? Une analyse du changement institutionnel à travers l'approche discursive
}

What does Ecologically Intensive Agriculture mean? An analysis of the process of institutionalization through discourse analysis

\section{Anne Musson et Damien Rousselière}

\section{(2) OpenEdition}

Journals

Édition électronique

URL : http://journals.openedition.org/economierurale/5037

DOI : 10.4000/economierurale.5037

ISSN : 2105-2581

\section{Éditeur}

Société Française d'Économie Rurale (SFER)

Édition imprimée

Date de publication : 1 novembre 2016

Pagination : $9-29$

ISSN : 0013-0559

Référence électronique

Anne Musson et Damien Rousselière, « De quoi l'agriculture écologiquement intensive est-elle le nom? Une analyse du changement institutionnel à travers l'approche discursive », Économie rurale [En ligne], 356 | novembre-décembre 2016, mis en ligne le 01 novembre 2018, consulté le 19 avril 2019. URL : http://journals.openedition.org/economierurale/5037; DOI : 10.4000/economierurale.5037 


\title{
De quoi l'agriculture écologiquement intensive est-elle le nom ? \\ Une analyse du changement institutionnel à travers l'approche discursive
}

\author{
Anne MUSSON • ESSCA, École de Management, Angers \\ anne.musson@essca.fr \\ Damien ROUSSELIĖRE • Smart-Lereco, Agrocampus Ouest, Angers \\ damien.rousseliere@agrocampus-ouest.fr
}

Analyser les processus de changement institutionnel en agriculture nécessite de comprendre les stratégies discursives des acteurs. Les auteurs s'intéressent dans cet article à l'évolution du concept d'Agriculture écologiquement intensive (AEI) à partir du corpus de presse regroupant l'ensemble des articles évoquant l'AEI depuis sa première apparition jusqu'en février 2014. L'analyse textuelle leur a permis d'identifier six classes de discours associés à l'AEI, d'observer une évolution chronologique de ces discours grâce à une caractérisation des classes par les années, de mettre en relief les jeux d'acteurs et d'apporter des éléments de réponse sur la question de l'institutionnalisation de I'AEI. Ils mettent en évidence un processus d'institutionnalisation qui semble se heurter, ces deux dernières années, à un détournement d'opportunité au profit du terme d'agro-écologie.

MOTS-CLÉS : agriculture écologiquement intensive, agro-écologie, analyse textuelle, changement institutionnel, détournement d'opportunité

\section{What does Ecologically Intensive Agriculture mean? An analysis of the process of institutionalization through discourse analysis}

Analyzing institutional change process in agriculture requires understanding discursive strategies of actors. This article study the evolution of the concept of Ecologically Intensive Agriculture (EIA), exploiting press corpus regarding all press articles about EIA since its first appearance to February 2014. Textual analysis identifies 6 kinds of discourses and highlights a chronological evolution. It emphasizes how actors play and how EIA is institutionalizing. We show the structuration of a new institutional fields braked since two years in favor of agro ecology concept. (JEL : B52, C38, Q1, Q5).

KEYWORDS: ecologically intensive agriculture, agro ecology, discourse analysis, textual analysis, institutional change

L a Conférence de Rio, en juin 1992, a donné naissance au concept de développement durable et demeure le symbole d'une remise en question d'un modèle de croissance non soutenable, de par les dommages environnementaux qu'elle provoque et son incapacité à réduire pauvreté et inégalités. Depuis trois décennies, malgré son ambiguïté et les controverses qui peuvent le parcourir (Vivien, 2005), le modèle de développement durable s'est institutionnalisé et la question de la «durabilité » a également fait évoluer nombre de paradigmes et de systèmes en place. Le mode de fonctionnement des exploitations agricoles a alors été remis en cause. Cette contestation du modèle agricole productiviste a stimulé nombre de réflexions et a abouti à plusieurs initiatives proposant des agricultures alternatives, telles que l'agriculture biologique, l'agro-écologie, l'agriculture raisonnée, l'agriculture à haute valeur environnementale, l'agriculture de conservation, et l'agriculture écologiquement 
intensive (AEI). Ces méthodes se sont diversement développées et ont connu des réussites variées dans la remise en cause du modèle « conventionnel $»^{1}$.

L'analyse de l'émergence de l'agriculture écologiquement intensive est un cas d'étude intéressant car, nouveau terme né en 2007 et signé de la plume de Michel Griffon (2007), il est, ces dernières années, entré dans le vocabulaire courant et exposé médiatiquement. Apparue dans un contexte de remise en question de l'institution que constitue l'agriculture « intensive et conventionnelle », l'agriculture écologiquement intensive se veut être un nouveau modèle de production. Par sa définition tout d'abord, l'AEI reposant «sur l'idée que les mécanismes naturels, ceux décrits par l'écologie, définie comme science, non comme activité politique, peuvent être amplifiés jusqu'à devenir presque exclusifs (ou dominants) en termes de pratiques agricoles $»^{2}$, et ce au bénéfice de la production alimentaire et des autres besoins de la société (Griffon, 2007, 2013a). L'AEI vise donc à maintenir le même rendement agricole que le modèle conventionnel, grâce à l'intensification écologique des fonctionnalités des écosystèmes, qui permet en parallèle la diminution des intrants artificiels chimiques (Griffon, 2007). Elle s'inscrit alors parfaitement dans le triptyque qui construit le développement durable : développement économique, respect de l'environnement et des préoccupations sociales. Autres singularités, cette agriculture «alternative», s'appuyant sur des pratiques plus anciennes (Goulet,

1. Le terme de conventionnel est utilisé faute de mieux, car il ne rend que peu justice à la diversité des modèles productifs qu'il peut désigner (De Raymond et Goulet, 2014). On notera d'ailleurs que cette unicité supposée d'un modèle conventionnel est au cœur des stratégies discursives de promoteurs de l'AEI.

2. http://www.aei-asso.org/fr/definir-les-agricultures/ (consulté le 10 février 2015).
2012), a été appropriée par des coopératives agricoles (Ghali et al., 2014) et s'est fortement développée dans le cadre d'un débat politique, celui du Grenelle de l'environnement (2007). Dès le départ, les acteurs classiques du changement institutionnel en agriculture sont déjà présents : les coopératives, les agriculteurs, les politiques et les citoyens. Le ministère français de l'Agriculture occupe ainsi une place particulière car il intervient sur la réglementation, le financement et l'appui au développement agricole (recherche et enseignement notamment).

Nous nous interrogeons sur la trajectoire d'institutionnalisation de l'AEI. Dans cet objectif, nous nous situons dans le cadre de l'analyse discursive des changements institutionnels et nous étudions l'ensemble des coupures de presse française concernant l'AEI. L'analyse textuelle nous permet d'identifier les types de discours associés à l'AEI et d'étudier leur évolution dans le temps. Cet article a ainsi pour objectif de répondre aux questions de recherche suivantes :

- Quels discours liés à l'agriculture écologiquement intensive peut-on identifier d'après les articles de presse ?

- Évoluent-ils dans le temps? Que nous apporte la caractérisation temporelle des différents types de discours au niveau méthodologique?

- Que nous enseignent l'identification des discours et leurs caractéristiques ${ }^{3}$ quant au processus d'institutionnalisation de l'AEI ?

L'analyse des changements institutionnels met de plus en plus l'accent sur les stratégies des acteurs à un niveau micro et leur capacité à faire évoluer des règles et les normes qui peu à peu peuvent se diffuser (Ostrom et Basurto, 2011). Dans ce

3. Acteurs, thématique, type de presse-locale, nationale, généraliste, spécialisée, intervalle de diffusion dans le temps (année concernée). 
processus de construction, comme le soulignent Suddaby et Greenwood (2005), le discours a une dimension performative et de légitimité : il contribue à la fois à la construction d'un cadre d'action et rend possible certaines pratiques. Notre article s'intéresse à cette dimension en l'appliquant au cas de l'agriculture. La littérature néo-institutionnelle s'attache à décrire les changements et les continuités des pratiques, et formes organisationnelles, en mettant notamment en avant le rôle et le positionnement des acteurs (DiMaggio et Powell, 1983 ; Scott, 1987 ; Ben Slimane, 2012 ; pour une revue de littérature, voir Pelletier, 2010), et s'intéresse spécialement à l'émergence de champs institutionnels (Greenwood et al., 2002). L'analyse des discours procure un cadre théorique utile pour comprendre la production sociale des phénomènes organisationnels et interorganisationnels (Philipps et al., 2004 ; Alvesson et Kärreman, 2000, Grant et al., 1998 ; Hardy et Phillips, 1999 ; Morgan et Sturdy, 2000 ; Mumby et Clair, 1997 ; Phillips et Hardy, 1997, 2002 ; Putnam et Fairhurst, 2001). Elle a pour objet de comprendre les institutions et leur construction, en étudiant les efforts discursifs des acteurs, qui deviennent des producteurs de récit et de discours. En particulier, les liens réciproques existant entre les discours, les textes et les actions peuvent être utilisés pour l'étude de l'institutionnalisation de concepts (Phillips et al., 2004). Ainsi, le travail institutionnel discursif repose sur l'utilisation du discours et permet aux acteurs d'influencer et d'agir sur les institutions (Ben Slimane, 2012). L'analyse discursive a donc pour objectif de comprendre l'émergence d'un concept institutionnalisé, étape importante dans le développement de la théorie institutionnelle (Lawrence et Phillips, 2004). En se situant dans ce cadre de l'analyse discursive, nous proposons d'analyser l'émergence et le développement du champ institutionnel de l'agriculture écologiquement intensive à travers une analyse textuelle des articles de presse le concernant.

Au sein de cet article, nous nous questionnons donc sur les discours liés à l'AEI, tels qu'ils apparaissent dans la presse: en quoi et comment évoluent-ils ? Notre contribution est double : l'analyse permet de décrire un processus d'institutionnalisation de l'AEI et démontre l'intérêt méthodologique de l'analyse textuelle longitudinale dans le cadre de l'approche discursive.

La section suivante situe notre étude dans un cadre théorique. L'analyse textuelle du corpus de presse et les résultats seront explicités dans la deuxième section. Enfin, nous discuterons dans la troisième section de l'implication de nos résultats quant à la trajectoire d'institutionnalisation de l'AEI et de l'apport de notre analyse au niveau méthodologique.

\section{Le processus discursif de l'institutionnalisation d'un concept}

Cette section cadre notre étude en deux temps. Nous décrivons tout d'abord le processus d'institutionnalisation et justifions ensuite l'utilisation de l'analyse des discours comme outil d'observation de ce processus.

\section{L'émergence de l'institutionnalisation du concept}

L'institutionnalisation est un phénomène ponctué d'événements particuliers mais il se caractérise principalement par un processus de théorisation (Rao et al., 2003), qui permettra de convaincre les acteurs concernés par l'innovation et ainsi, de la diffuser. Dès lors, comprendre comment émerge un concept qui, ensuite, s'institutionnalise s'avère nécessaire à l'étude de son processus d'institutionnalisation (Lawrence et Phillips, 2004).

L'émergence prend naissance dans un fait particulier, pouvant être qualifié de 
«secousse » et prendre la forme d'une rupture technologique, d'un soulèvement social, d'une nouvelle réglementation (Brulé et Audebrand, 2009). Elle peut aussi se caractériser par une rupture paradigmatique : la mise en exergue des principes de développement durable depuis la Conférence de Rio en 1992 a progressivement amené les professionnels de l'agriculture à remettre en question leurs pratiques, à la lumière de leurs externalités sociales et environnementales, bien en amont de l'apparition du terme d'agriculture écologiquement intensive. Ainsi, le développement de l'AEI prend racine bien avant qu'en soient posées les bases et une définition ${ }^{4}$. Si l'institutionnalisation est un processus social le long duquel les individus partagent une même définition de la réalité sociale (Phillips et al., 2004), il n'est donc pas porté par un seul acteur, mais par un ensemble d'acteurs, qui parfois s'opposent (Lawrence et Suddaby, 2006). Brulé et Audebrand (2009) identifient deux phases principales constituant le processus d'appropriation d'une innovation.

Dans un premier temps, l'ancrage va consister à connecter l'innovation à une technologie ou à un concept connu ou reconnu; en mettant en avant ce qui la différencie ou ce qui l'apparente, selon les intérêts des acteurs. Si l'AEI s'inscrit dans le paradigme du développement durable, ses promoteurs pourraient ainsi mettre en avant le respect des trois piliers (rentabilité économique, diminution de l'impact environnemental et prise en compte de l'aspect social), pendant que d'autres acteurs auraient la possibilité de minimiser, voir remettre en cause, la prise en compte de ces principes et d'invoquer la prédominance d'intérêts particuliers, lobbyistes ou politiques. Cette première phase

4. Les techniques auxquelles l'AEI peut se référer (techniques sans labour, utilisation des auxiliaires de culture, etc.) sont établies antérieurement. de l'ancrage ou catégorisation est ensuite suivie de l'intégration. Il s'agit alors de situer l'innovation par rapport au cadre : la situer par rapport quelque chose de connu, et ensuite la spécifier. Ainsi, l'AEI n'est pas synonyme du développement durable, puisqu'elle s'intéresse aux problématiques d'un secteur particulier et précis ; dans le même temps, elle se différencie également et clairement de «l'agriculture conventionnelle ».

Dans un second temps, l'objectivation est une phase proche du processus de concrétisation. Cette phase va isoler et dessiner le concept (Doise, 1993), lui attribuer des caractéristiques singulières et partagées et permettre son appropriation par les acteurs (Moscovici, 1976). Il s'agit alors de rendre l'agriculture écologiquement intensive concrète: quelles en sont les techniques? Sont-elles appliquées dans certaines exploitations ? Les résultats attendus sont-ils vérifiés et exposés ? Au cours de cette phase, les acteurs de l'AEI s'approprient le concept et une définition partagée doit progressivement s'imposer.

Le développement du concept est donc le terrain de jeux d'acteurs, aisément qualifiables d'entrepreneurs institutionnels, dont la capacité de certains à critiquer le cadre institutionnel existant et la stratégie de réponse des autres vont largement influencer la forme de changement institutionnel (McInerney, 2013). Le changement institutionnel, comme le soulignent Maguire et Hardy (2009), conduit à la fois à un long processus de désinstitutionnalisation des pratiques précédentes et à un processus d'institutionnalisation des nouvelles pratiques. Pour qu'un concept s'institutionnalise, les entrepreneurs institutionnels doivent « influencer les cadres législatifs ou réglementaires, affecter les normes culturelles et les valeurs ou établir des structures ou des processus considérés comme acquis » (Lawrence, 1999, dans Zilber, 2007). Ainsi, si l'agriculture 
écologiquement intensive se révèle comme un concept s'institutionnalisant, elle est nécessairement l'œuvre d'entrepreneurs institutionnels, qui peuvent être par exemple des coopératives agricoles, des groupements de citoyens, des lobbys ou des partis politiques, influençant avec succès les normes et les pratiques établies au sein de «l'agriculture conventionnelle », et de fait, les acteurs s'y rattachant.

\section{Le rôle de l'analyse des discours dans le processus d'institutionnalisation}

L'approche discursive de l'institutionnalisation (Phillips et al., 2004) propose un cadre théorique pour étudier l'institutionnalisation du concept d'agriculture écologiquement intensive à travers les articles de presse française. Zilber (2007) formalise les processus d'institutionnalisation de la manière suivante. Les textes, qui, au sens large, peuvent aussi être qualifiés de discours, par la prise de distance réflexive et en conceptualisant les actions, fournissent des bases informationnelles qui mettent en forme les actions futures et participent ainsi à un processus d'institutionnalisation (Munir et Phillips, 2005). Ceux-ci diffusent une compréhension et du sens commun qui vont dessiner ce qui forme une institution, notamment des pratiques et des pensées communément établies et partagées (Zilber, 2007). Les acteurs deviennent des entrepreneurs institutionnels lorsqu'ils sont porteurs de ces textes (Phillips et al., 2004).

Phillips et al. (2004) ont initié un courant de recherche qui considère l'analyse des discours comme un cadre cohérent pour le processus d'institutionnalisation (voir également Ben Slimane, 2012 ; Hardy et Maguire, 2008 ; Phillips et Malhotra, 2008). C'est en effet à travers les textes que l'information à propos de l'action est largement diffusée et va alors influencer les autres (Phillips et al., 2004) ; c'est notamment pourquoi l'analyse des coupures de presse nous semble particulièrement cohérente. De plus, celles-ci, en tant que discours macro-culturel ${ }^{5}$, prennent largement part au contexte au sein duquel un concept émerge (Lawrence et Phillips, 2004). Les articles de presse sont particulièrement intéressants car ils sont facilement accessibles et le plus souvent légitimés par les participants à la notion émergente, ce qui leur confère un impact conséquent sur l'émergence du concept (Lawrence et Phillips, 2004). Ils présentent également l'ensemble des caractéristiques des actions menant à la production de discours institutionnalisant identifié dans le modèle discursif présenté par Phillips et al. (2004, voir précisément schéma 2) : le producteur de texte est légitimé, a une certaine autorité ; le corpus est cohérent et structuré ; les discours se produisent progressivement, au fur et à mesure que les textes vont du local au global.

Selon la typologie de Ben Slimane (2012), les acteurs peuvent créer des opportunités, les contrer, les déplacer ou les détourner. L'analyse des coupures de presse peut permettre d'identifier des conflits d'institutionnalisation entre les acteurs. Ainsi, contrer des opportunités qui se basent sur la contre-production de texte (Ben Slimane, 2012 ; Maguire et Hardy, 2009).

\section{Analyse discursive longitudinale de l'agriculture écologiquement intensive à travers les articles de presse}

\section{Données}

Pour les besoins de l'analyse textuelle, nous avons rassemblé l'intégralité des articles de presse mentionnant «agriculture écologiquement intensive », répertoriés

5. Le discours macro-culturel se compose de l'ensemble des discours et des institutions associées qui s'étendent le long d'une frontière, et qui sont largement compris puis admis dans une société (Lawrence et Philipps, 2004). 
par la base Factiva jusqu'au mois de février 2014. Une autre possibilité serait de s'appuyer sur d'autres corpus, comme le fait Ben Slimane (2012), sur l'analyse des discours d'un acteur en particulier, sur l'analyse des rapports d'activité (Rousselière et Vézina, 2009), sur celle des sites Internet (Michaud, 2013) ou encore des entretiens réalisés auprès des acteurs (Musson, 2012). Ces différentes possibilités ne permettraient pas de couvrir l'émergence du concept ainsi que la diversité des approches. Tous les acteurs n'ont pas, en effet, pris position sur le sujet de manière explicite. L'association pour le développement de l'AEI n'existe que depuis 2010, ce qui impliquerait la perte de toute la partie préalable. L'analyse de discours d'entretiens avec les acteurs contient quant à elle le risque de la rationalisation a posteriori (Bourdieu, 1986), comme l'a bien montré Reynaud (2001) en matière d'analyse de stratégies d'entreprise. Notre approche se base sur une analyse exhaustive de l'ensemble des coupures de presse, qui sont les seuls documents publics faisant référence à l'AEI depuis l'émergence de ce terme.
Le corpus se compose donc de 473 articles, le premier datant du 6 mars 1998 et le dernier du 27 février 2014. Seulement 15 articles sont parus avant 2006, ainsi, par souci de cohérence et de lisibilité, nous regrouperons ceux-ci ainsi que les parutions de 2006 sous la même variable «<2007 ». Le graphique 1 décrit notre corpus en fonction des variables discriminant les articles et qui caractériseront les discours issus de l'analyse textuelle. Notre corpus se dessine selon trois caractéristiques : la publication dans une presse locale ou nationale, la publication dans une presse générale ou économique/technique et la date de publication. Nous remarquons ainsi une envolée des parutions à partir de 2010. En 2013, le nombre d'articles est inférieur à 2012, mais, concernant 2014, ne sont comptabilisées que les parutions de janvier et février, et si la tendance se prolonge, 2014 sera l'année durant laquelle le nombre d'articles concernant l'AEI est le plus élevé (voir projection 2014 sur le graphique 1.2.) Une forte majorité (69\%) des articles sont parus dans la presse locale (31\% dans la presse nationale) et

\section{Graphique 1. Caractéristiques du corpus d'articles de presse}

\section{Graphique 1.1. : Description de l'échantillon selon le type de presse : locale/nationale ; générale/spécialisée}

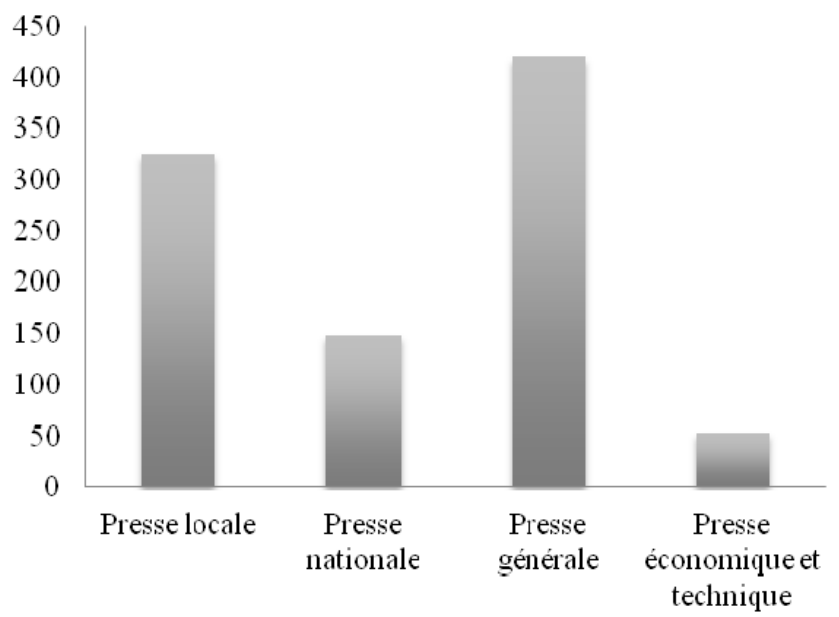




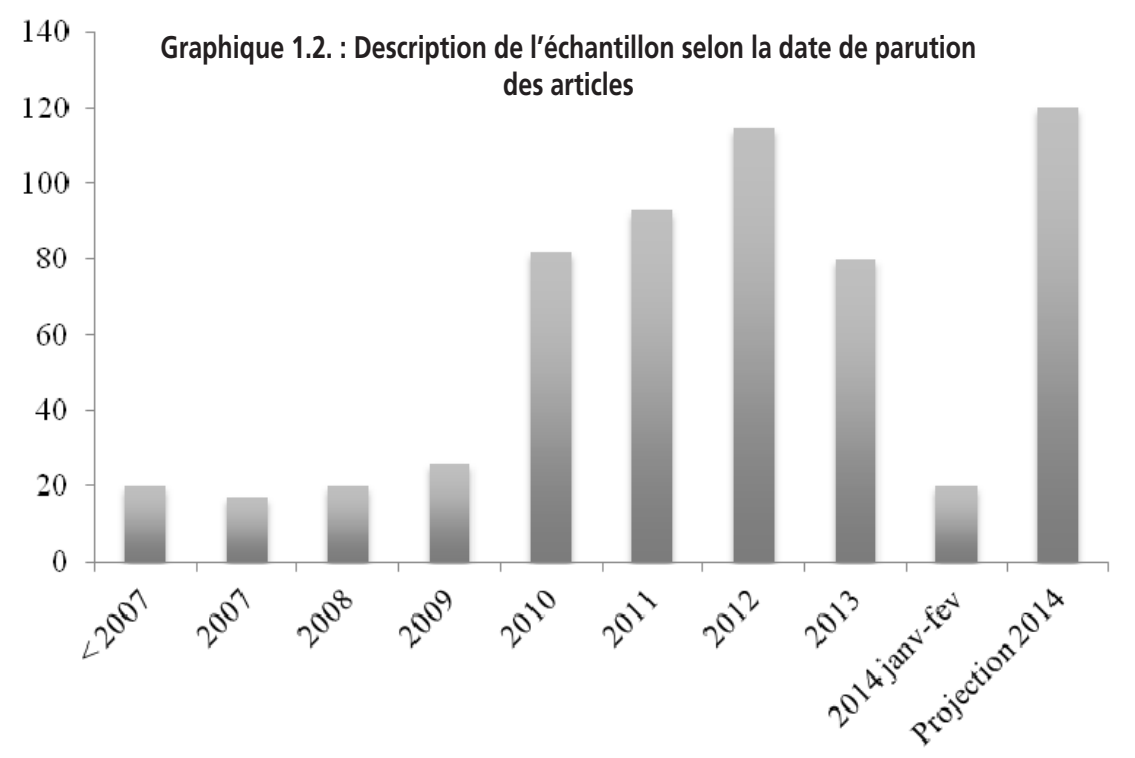

Source : Factiva, données par les auteurs.

encore davantage dans la presse généraliste $(89 \%)$.

Le corpus d'articles de presse semble présenter l'ensemble des caractéristiques énoncées par Phillips et al. (2004). Ainsi, tant qu'il s'agit d'une presse régulière, à large diffusion, les sources sont «légitimes », « reconnues ». Si l'on peut soupçonner, à juste titre, un biais de subjectivité lié à la presse, les coupures utilisant beaucoup d'interviews, présentant des études de cas et commentant des actions mises en place, prétendent ainsi à une certaine objectivité. À ce titre, l'ensemble est cohérent: les techniques présentées sont robustes, validées scientifiquement mais aussi sur le terrain par des agriculteurs et le concept est politiquement valorisé, du niveau local au niveau de l'État. Enfin, la multiplicité des sources permet également de tempérer l'éventuel biais lié à la subjectivité de certains journaux.

\section{Méthodologie}

Nous avons ensuite choisi de traiter les 473 articles de presse à l'aide du logiciel
Alceste $^{6}$ (version 2010) afin de réaliser une analyse textuelle du corpus (Reinert, 1993). Ce logiciel, dont l'acronyme signifie «analyse des lexèmes co-occurents dans les énoncés simples d'un texte », met l'accent sur les ressemblances et les dissemblances de vocabulaire. Au sein d'un corpus, c'est-à-dire l'ensemble des textes réunis pour l'analyse, il permet de repérer la présence d'ensembles d'énoncés, appelés classes, qui mobilisent des vocables similaires dans des classes sémantiques. La significativité du vocabulaire partagé au sein de cette classe est évaluée à l'aide d'un test du chi-deux. Alceste fonctionne sur la base d'une double classification descendante hiérarchique, procède par partitions successives du corpus et met ainsi au jour des classes d'énoncés (Helme-Guizon et Gavard-Perret, 2004), qui décrivent ainsi des profils différents. Elles se définissent grâce notamment à leur vocabulaire spécifique et les variables illustratives qui leur

6. http://www.image-zafar.com/index_alceste. $\mathrm{htm}$ 
sont attachées (Capdevielle-Mougnibas et al., 2004).

Nous testons plusieurs discriminants sur les classes qui vont émerger de l'analyse textuelle : l'année de publication, la dimension locale ou nationale du journal ayant publié l'article et son caractère spécialisé ou généraliste. Nous nous demandons par exemple si la représentation de l'agriculture écologiquement intensive et les types de publication évoluent au cours $\mathrm{du}$ temps. Nous discriminons également les articles selon leur parution dans la presse locale ou nationale, pour observer l'évolution des discours selon cette caractéristique. Nous cherchons en effet à savoir si les textes vont du local au global, tel que décrit au sein du modèle discursif présenté par Phillips et al. (2004). Ces informations sur les articles (dates et type de journal), appelées « mots étoilés », ou « variables illustratives », caractérisent, pour le logiciel, les unités de contextes initiales (u.c.i.), qui correspondent aux articles (un article $=$ une u.c.i.). On peut alors identifier les attributs des articles qui caractérisent certaines classes - une année, par exemple. En raison du faible nombre d'articles par mois, et de leur forte saisonnalité ${ }^{7}$, nous choisissons d'avoir une approche discrète du processus temporel. Le temps est ici inséré en tant que variable catégorielle.

Notre approche longitudinale à une étape est donc originale au regard de l'utilisation d'Alceste dans la littérature en sciences de gestion (e.g. Chahine et Filatotchev 2008; Illia et al., 2014) et se distingue de celle à deux étapes proposée par Chekkar et Ornée (2006) qui comparent une analyse thématique et une analyse chronologique afin de caractériser des périodes. Enfin, elle se différencie de celle de Geka et Dargentas (2010) qui codifient a priori les périodes temporelles pour ensuite voir si elles sont caractéristiques de

7. Environ $20 \%$ des articles sont publiés en juin et seulement 2 à $3 \%$ en juillet en août. certaines classes. La classification a posteriori, proposée ici, et non a priori s'avère davantage pertinente concernant l'étude d'un phénomène émergent.

\section{Résultats}

L'analyse textuelle a donc permis d'identifier des classes qui décrivent des représentations différentes de l'agriculture écologiquement intensive, représentées sur le graphique 2.

Ce sont ces classes qui sont représentées par des cercles de couleur, la forme de ceux-ci étant dictés par le positionnement du discours par rapport aux axes du graphique Les expressions attribuées aux axes (en italique, de police noire et de taille moyenne) sont déterminées par interprétation (Reinert, 1993), au vu du positionnement des mots caractéristiques sur le graphique. Lorsque les cercles se superposent, et en présence d'une bonne représentation de l'inertie totale ${ }^{8}$, cela signifie qu'une partie du discours est «commune » à différentes classes. Les mots (en italique, de police noire et petite) sont représentatifs du discours de chaque classe et succèdent à l'appellation de la classe. Par exemple, sur le graphique 2, la classe 3, appelée «nouvelles techniques » a pour caractéristique d'utiliser fortement et par ordre d'importance, les mots suivants : engrais, chimie, sol, plante, rendement, eau, pesticides, etc. Suivant l'axe vertical, ce discours s'attache à décrire l'AEI comme une démarche au sein des exploitations, et suivant l'axe horizontal, il s'intéresse davantage aux actions liées à l'AEI plutôt qu'au discours que cette démarche véhicule, ou à la communication nécessaire pour l'expliquer.

8. Les classes sont étudiées et décrites à la lumière de l'analyse factorielle en coordonnées, projetée graphiquement selon deux axes présentant un pourcentage d'inertie de $55 \%$ (graphique 2), de la classification descendante hiérarchique et du vocabulaire caractéristique de chaque classe (annexe 1), et, enfin, suivant leurs caractéristiques issues des variables illustratives (tableau 1). 
RECHERCHE

Anne MUSSON, Damien ROUSSELIÈRE

Graphique 2. Analyse factorielle en coordonnées. Représentations de l'AEI à travers les coupures de presse

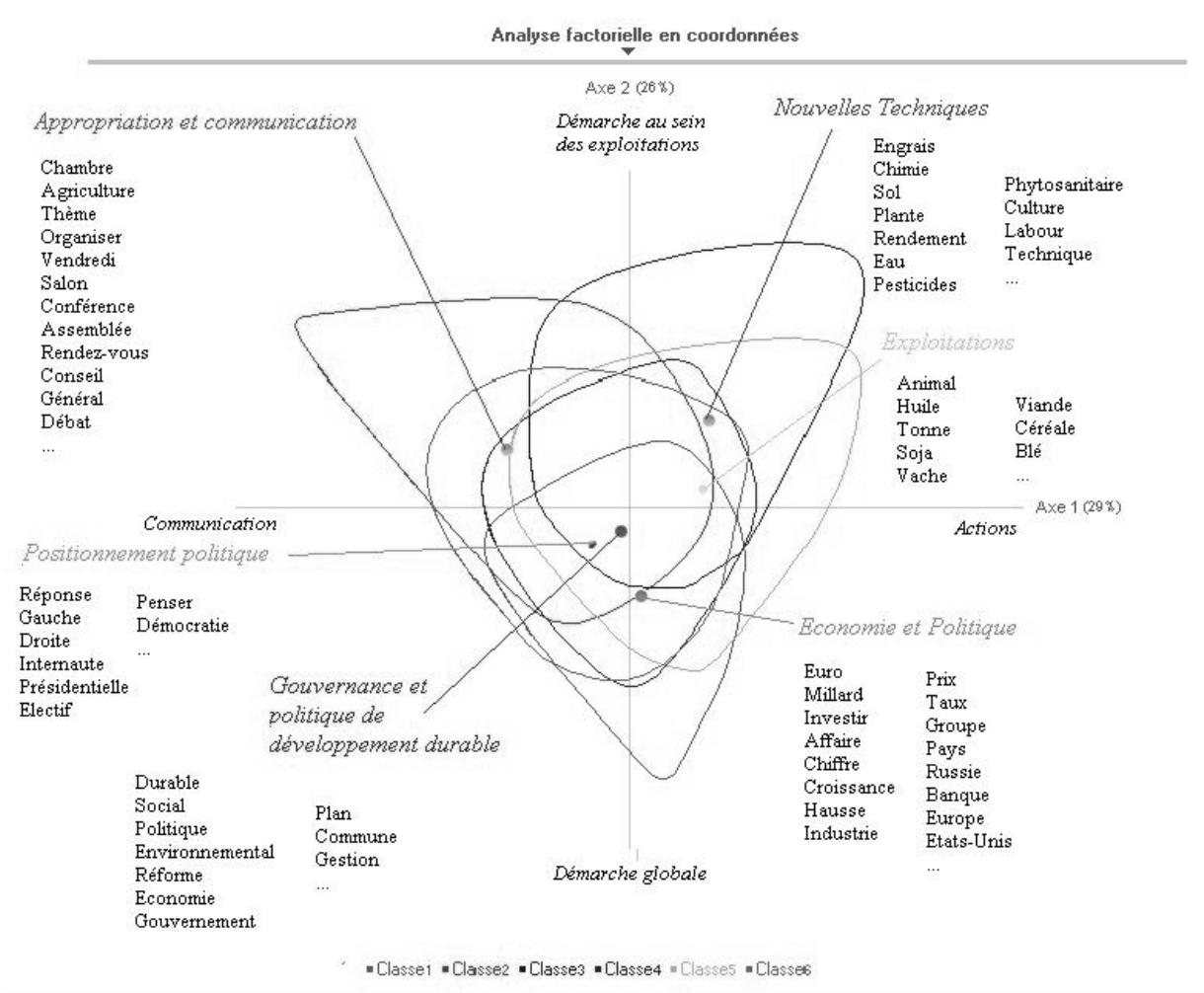

Source : Graphique en couleur disponible sur demande aux auteurs.

À l'issue de l'analyse, nous obtenons 6 classes représentant $68 \%$ du corpus (graphique 2). Suivant la classification descendante hiérarchique, les classes 1 et 4 , toutes deux liées au discours politique, sont proches (voir dendrogramme, annexe 1). Le tableau 1 récapitule les variables supplémentaires caractéristiques de chacune des classes (par leur présence et leur absence). La classe 1 comprend $26 \%$ des énoncés classés, qui proviennent essentiellement d'articles parus dans la presse nationale, économique et technique, principalement en 2006 et 2013. Ce groupe, appelé « économie et politique », s'intéresse au rendement économique, qui est l'un des piliers de l'AEI, à travers un vocabulaire tel qu' « euro », « milliard », « investir », «chiffre», «prix », etc.; et replace le concept dans un contexte général de croissance verte (« croissance », « industrie », « éolien », « entreprise », « marche », etc.) et de compétitivité internationale (« américain », « groupe », « Russie », « dollar», « client »). La classe 4 présente un discours qui a une portée plus globale et renvoie plus spécifiquement à une dimension politique que la classe 1. Il s'intéresse à la mise en place de politique de développement durable au sein desquelles l'AEI semble avoir sa place ${ }^{9}$. Le vocabulaire est alors lié au développement durable (« durable»,

9. Le lien se dessine dans les deux sens : l'agriculture est un domaine d'application du développement durable au sein de laquelle l'AEI est présente; le développement durable est une dimension de l'AEI. 
« social », « environnement», « économie », « équitable », « responsabilité »), à la politique et ses outils ("politique», « réforme », « gouvernement», «plan», «gestion», « fiscal») et au territoire (« commune », « européen »). Cette classe représente $18 \%$ du discours classé, issu principalement de presse nationale, économique et technique, dont les articles sont parus, pour la plupart, en 2014 et 2008. La classe 6 , représente $8 \%$ des discours classés. Elle est proche des classes 4 et 1 en envisageant l'AEI sous l'angle politique. Cependant, le discours est beaucoup plus spécifique : le cercle s'étend davantage vers la gauche du graphique 2, ce qui est caractéristique d'une représentation liée à la communication. Il s'agit en fait ici de placer l'AEI au cœur du débat politique. Le vocabulaire utilisé est alors très spécifique : « gauche », « droite», « présidentielle», « électif», « démocratie », etc. Le groupe «positionnement politique » est une représentation à l'origine de la diffusion du terme de l'AEI, puisque les articles représentés sont issus de la presse nationale datant de 2006 et 2007 . Le discours propre à la classe 2 apparaît plus tard (2011-2012), il est caractéristique de la presse locale et se situe encore plus à gauche sur le graphique 2 : c'est la classe de l' « appropriation et [la] communication », qui regroupe $19 \%$ du discours classé. On découvre ici un vocabulaire lié à l'événementiel et l'organisation ( «thème », « organiser », « salon », « conférence », « assemblée », « rendez-vous », « débat», « journée », « rencontre », « association »), et ceci au niveau local («chambre d'agriculture », « conseil général », et encore «association » et « rendez-vous »). Le discours propre à cette classe semble nous décrire la diffusion de l'AEI, que l'on a fait découvrir aux agriculteurs (et, plus largement, au grand public) grâce à des organismes et associations locales, dont les actions sont relayées aux acteurs du territoire concerné à travers ces articles de presse. Enfin, les classes 3 et 5 ont en commun de s'intéresser aux démarches concrètes au sein des exploitations liées à l'AEI (leurs cercles s'étirent vers le haut et la droite du graphique 2). Ainsi, la classe 5 , qui regroupe $11 \%$ du discours classé, s'intéresse à l'application de l'AEI selon les types d'exploitations et de cultures concernées par l'AEI ( « animal », « soja », « vache », « viande », « céréales », «blé », « fraise », etc.) et dans quelle(s) mesure(s) («tonne », « hectare », « litre »). La classe 3, correspondant à $18 \%$ du discours classé, décrit plus précisément les nouvelles techniques mobilisées au sein de l'AEI, et en évoque les impacts aussi bien en termes d'utilisation d' « engrais », d' « eau », de «pesticides », de «phytosanitaires », d' « intrants », de nouvelles méthodes (« sol », « labour», « biologie, « technique », « naturel », « réduire, « intrants ») qu'en termes de « rendement». Ces deux classes nous décrivent le passage d'une technique à une autre, et tendent à expliquer un ensemble de pratiques et de techniques agronomiques constitutives de l'AEI. Ces discours, qui peuvent être techniques, s'adressent cependant à tous, car ils sont caractéristiques d'une presse généraliste. La classe 5 diffuse son discours en 2007, 2009 puis 2013, pendant que le discours propre à la classe 3 apparaît en 2008, 2010, 2012.

Le discriminant temporel apparaît à chaque fois comme une caractéristique significative des classes. Nous identifions une progression chronologique dans les types de discours liés à l'AEI. En effet, à la naissance du concept, le discours est surtout politique et s'inscrit dans une démarche globale liée au développement durable. En effet, avant 2008, les articles parus s'inscrivent dans les classes 1 et 6 , «économie et politique » et «positionnement politique ». Il s'agit plutôt d'une presse spécialisée et nationale. Dans un second temps, entre 2007 et 2010, le terme se diffuse dans la presse généraliste, mais 


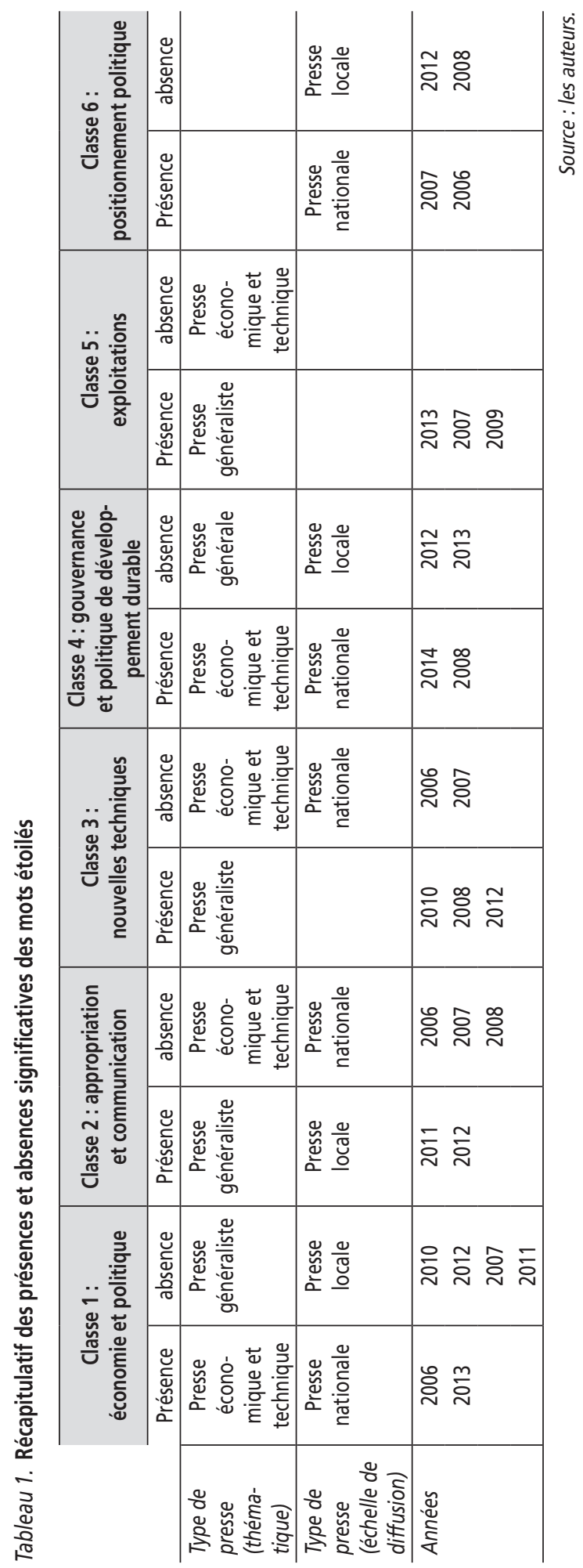


avec des descriptions concrètes et techniques. Les discours liés aux classes 3 et 5 (« nouvelles techniques » et «exploitations ») semblent avoir contribué à asseoir les fondements de l'AEI, en en expliquant précisément les techniques et en en décrivant ses applications. Puis, en 2011-2012, la classe 2 ( « appropriation et communication ») apparaît prépondérante. Le terme et le concept se diffusent et s'expriment alors au niveau local. Ces deux dernières années, le discours politique semble faire son retour, notamment à travers la classe 4 ( "gouvernance et politiques de développement durable »), dans une presse nationale et spécialisée. Au niveau de la presse générale, le discours de la classe « exploitations » est toujours présent (2013), comme celui des «nouvelles techniques » (2012). Ces articles persistent dans la justification du terme et de son existence, à travers des explications aussi précises que concrètes. Le retour à la presse nationale semble nous indiquer que le concept aurait été approprié par les spécialistes et/ou au niveau local, pendant que la diffusion du terme est encore en cours pour le grand public.

\section{La chronologie des discours et son lien avec les pratiques}

L'analyse textuelle longitudinale nous permet ainsi de proposer une périodisation des discours en plusieurs temps. Les quatre périodes s'interprètent de la façon suivante.

\section{Les origines de I'AEI (1998 à 2006-2007) et démarche de développement durable}

La première période est celle des origines du concept de l'AEI. Elle correspond à la remise en cause de l'agriculture intensive. En parallèle, se développe une définition de l'AEI par Michel Griffon, chercheur au CIRAD. Ce terme rencontre les préoccupations d'une coopérative (Terrena) qui doit redéfinir son projet politique (à la suite de la fusion entre deux coopératives d'origines différentes et le positionnement de la nouvelle entité par rapport aux enjeux environnementaux). Le contexte politique est celui préparatoire au Grenelle de l'environnement. Ce contexte apparaît comme étant celui donnant le sens à l'émergence de ce nouveau terme. Il s'agit de trouver un compromis «gagnant-gagnant» de ce que Griffon à la suite de Conway (1998) a nommé : une « révolution doublement verte ».

\section{Institutionnalisation et exemplarité (2008-2010)}

La deuxième période est celle du début de l'institutionnalisation du concept et celle de la proposition de l'AEI en tant que solution aux problèmes de développement durable appliqué au secteur agricole. Deux événements majeurs sont à souligner pendant cette période : la création des Terrenales (qui ont eu lieu pour la $1^{\text {re }}$ fois en 2010) et la constitution de l'Association internationale pour le développement de l'AEI (janvier 2010). Cette association se constitue en tant que lobby. Ces événements s'inscrivent dans une volonté de diffuser «par l'exemple » le concept. Les verbatim suivants extraits de notre base de données d'article de presse illustrent cette proposition.

«Jeudi et vendredi derniers, dans un laboratoire géant et en plein air, Terrena a présenté les résultats probants des travaux menés avec des agronomes, dont Michel griffon, directeur adjoint de l'agence nationale pour la recherche et défenseur de l'AEI, des scientifiques, mais aussi des agriculteurs. » (2010)

«Ainsi, on redécouvre les bienfaits de la couverture des sols par différentes espèces végétales dont certaines fixent l'azote de l'air nécessaire au développement des autres. Ce qui remplace les engrais, mais aussi protège les sols et augmente le stockage de l'eau. » (2010) 
RECHERCHE

«La nutrition animale n'est pas en reste. Des huiles essentielles, notamment de la cannelle, servent de complément alimentaire aux volailles pour renforcer leurs défenses immunitaires. Résultat: 90 des lots testés chez 357 éleveurs ne sont plus traites par antibiotiques. Des essais sont en cours sur les lapins, les ruminants et les porcs. » (2008)

\section{Les acteurs locaux de I'AEI (2011-2012)}

La période suivante correspond à une période où de nouveaux acteurs s'approprient la diffusion du concept opéré par Terrena, cette dernière s'affirmant comme étant le principal pôle. Ainsi, on note la refonte de la communication de Terrena autour de la bannière de l'AEI et le renouvellement de l'événement des Terrenales avec la mise en avant des «sentinelles de la Terre », coopérateurs exemplaires de Terrena (notamment dans le cadre d'un ouvrage où sont exposées les techniques expérimentées chez leurs adhérents).

La création de la Chaire AEI formalise les relations entre Terrena, les deux autres grandes coopératives agricoles (Triskelia et Agrial) et les trois établissements de l'enseignement supérieur agricole de l'Ouest de la France. La Chaire a pour objectif de financer des travaux de recherche sur l'applicabilité de l'AEI et des stages de formation en agro-écologie pour les techniciens des coopératives.

«Ce mercredi, Stéphane Le Foll est attendu à l'École supérieure d'agriculture, ESA, à Angers. Le ministre de l'Agriculture clôturera la $3^{e}$ édition des entretiens de l'agriculture écologiquement intensive.» (2012)

«Jusqu'au 22 juin, dix agriculteurs $d u$ Finistère ouvrent leurs portes dans le cadre de la semaine de l'innovation en Bretagne organisée par la Chambre d'agriculture. Une occasion pour découvrir de nouveaux savoir-faire, éventuelle source d'inspiration pour d'autres exploitations. Les rendez-vous innov' action mettent l'agriculture écologiquement intensive, AEI, au centre des échanges. » (2012)

\section{Vers l'agro-écologie comme politique publique ? (2013-2014)}

La dernière période semble être marquée par l'émergence importante du ministère (français) de l'agriculture porteur d'un nouveau concept (agro-écologie), acteur déjà présent aux périodes précédentes et qui devient actif en préparant la Loi d'avenir agricole. En effet, les premières phrases de la loi font explicitement référence à l'agro-écologie ${ }^{10}$ :

«Le ministre de l'Agriculture, de l'agroalimentaire et de la forêt a présenté un projet de loi d'avenir pour l'agriculture, l'alimentation et la forêt. L'agriculture française et les secteurs agroalimentaires et forestiers doivent relever le défi de la compétitivité pour conserver une place de premier plan au niveau international, et contribuer au développement productif de la France. Ils doivent continuer à assurer une production alimentaire de haut niveau qualitatif et en quantité suffisante face à l'augmentation de la population mondiale, tout en s'inscrivant dans la transition écologique. Le projet agro-écologique pour la France a ainsi pour objectif de placer la double performance économique et environnementale au cœur de pratiques agricoles innovantes ${ }^{11}$.»

Durant la même période, le numéro spécial 2013-1 de la revue Projet présente à la fois un article de Stéphane Le Foll (Le Foll, 2013), identifié en tant

10. Le projet de loi d'avenir pour l'agriculture ne fait pas partie de notre corpus mais nous en extrayons les premières phrases à titre d'illustration, car elles reprennent et reformulent dans un cadre d'agro-écologie les principaux thèmes de l'AEI.

11. http://www.legifrance.gouv.fr/affichLoiPreparation.do;jsessionid $=2559 \mathrm{~A} 3 \mathrm{BE} 1 \mathrm{~A} 613580 \mathrm{~F} 78$ 9267A3BAE30CD.tpdjo17v_1?idDocument=JO RFDOLE000028196878\&type $=$ general 
que ministre de l'Agriculture, qu'un article de Michel Griffon (Griffon, 2013b). L'éditorial de Jean Merckaert intitulé «Polyphonie pastorale » situe ce numéro explicitement dans le cadre de la négociation de la nouvelle Politique agricole commune (PAC) (Merckaert, 2013). La chronologie des événements concernant la $\mathrm{PAC}$, telle que proposée par le ministère français de l'Agriculture, montre bien que la Loi d'avenir agricole se situe au cœur de nombreuses actualités européennes (annexe 2). Il semblerait effectivement qu'elle ait été travaillée en parallèle des réflexions autour de la PAC.

Le graphique 3, établi à partir des coupures de presse de la même base de données, met bien en évidence la dynamique nouvelle du terme «agro-écologie» au regard du terme «agriculture écologiquement intensive ».

L'étude de l'évolution temporelle des discours à travers l'analyse textuelle met en évidence un processus d'institutionnalisation tel que nous l'avons décrit dans la première partie de cet article :
- L'émergence du concept prend appui sur la rupture paradigmatique du développement économique impulsé par la Conférence de Rio en 1992.

- On observe dans un premier temps le processus d'ancrage de l'AEI au paradigme du développement durable (2006/2007).

- L'objectivation a ensuite dessiné le concept : les techniques de l'AEI sont décrites, démontrées scientifiquement et mise en pratique et en scène (via les Terrenales) au sein des exploitations. Les acteurs institutionnels apparaissent clairement et se positionnent par rapport à l'AEI : l'association pour une agriculture écologiquement intensive, les coopératives et surtout Terrena, les chercheurs, les agriculteurs, les chambres consulaires, les leaders de pensées, les associations de citoyens et également les responsables politiques locaux. À la fin de cette phase (2008-2012), la définition de l'AEI telle qu'exposée par Michel Griffon s'est imposée et est partagée. Chacun peut en effet identifier ce

\section{Graphique 3. Évolution respective du nombre d'articles de presse mentionnant l'AEl ou l'agro-écologie}

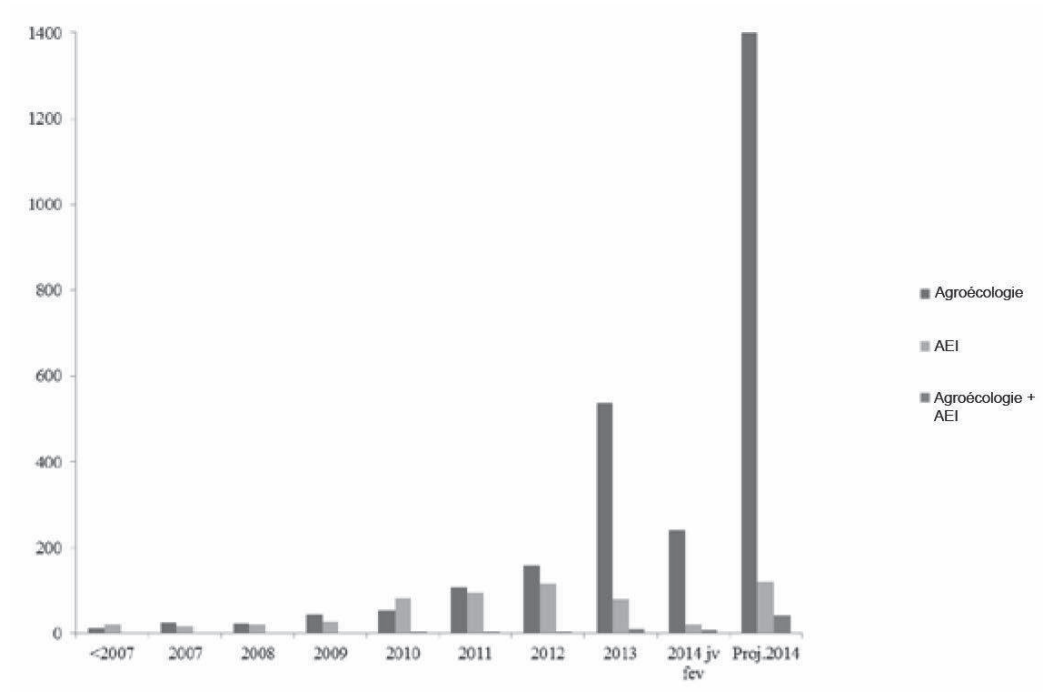

Note : compte réalisé au $1^{\mathrm{er}}$ mars 2014 par les auteurs. 
qu'est précisément l'AEI. On observe également un passage des articles de presse du national (première et dernière période) au local (périodes intermédiaires).

En revanche, la dernière période (2013/2014) sonne comme un frein à l'AEI comme institution. Il est probable qu'apparaisse alors un détournement d'opportunité, par l'acteur politique national, reprenant à son compte l'institutionnalisation de l'AEI en lui apposant le terme d'agro-écologie. Les techniques de l'AEI et de l'agro-écologie étant les mêmes, elles sont d'ores et déjà décrites, prouvées, et la définition en est partagée. Mais c'est le terme agro-écologie qui semble prendre le pas, à partir de 2012 (voir graphique 3), cette tendance étant autant symbolisée que stimulée par la loi d'avenir agricole présentée en janvier 2014. Elle a ainsi présenté l'agro-écologie comme ligne directrice et elle fut, et est encore aujourd'hui, largement relayée par les médias, tant généralistes que spécialistes. Si « détourner une opportunité de changement a pour objectif de libérer des ressources dans le champ mais d'en empêcher l'acquisition par les challengers » (Ben Slimane, 2012), il est possible que le champ institutionnel de l'agriculture écologiquement intensive soit récupéré politiquement au niveau national, mais sous l'appellation « agro-écologie », celle-ci n'étant pas comme l'AEI associé aux coopératives, notamment Terrena. Ce détournement d'opportunité s'inscrit en effet dans le cadre des débats initiés en 2013 sur la mise en place d'une nouvelle politique agricole commune (PAC) axée sur un «verdissement» (Allaire et al., 2013). L'agro-écologie est évoquée par le ministre comme la priorité pour la mise en place du deuxième pilier de la PAC en France (Le Foll, 2013). Il est toutefois nécessaire pour un acteur public de reformuler le débat en utilisant un terme moins facilement identifiable à un acteur privé spécifique. Le terme agro-écologie, qui bien que polysémique, comporte une forte légitimité scientifique (e.g. ErnestoMendez et al., 2013 ; Wezel et al., 2014), et présente a priori cette qualité.

L'acteur «Union européenne », par le biais de la PAC, semble donc également impliqué dans ce détournement d'opportunité. En effet, le graphique 3 montre l'ascension du terme d'agro-écologie, relativement à l'AEI, commence à faire le poids avec AEI à partir de 2011. En parallèle, la chronologie des actualités liées à la PAC tels que rapportée par le ministère ${ }^{12}$ montre que le premier événement majeur a lieu en octobre 2011 (proposition de réforme de la PAC). À partir de 2013, les actualités se multiplient et le terme d'agro-écologie prend l'ascendant sur celui d'agriculture écologiquement intensive (graphique 3).

Il est intéressant de noter le développement parallèle par Terrena d'une marque, labellisée Nouvelle Agriculture, qui pour l'instant ne fait référence qu'au terme d'AEI et non à l'agro-écologie. Suivant le destin du terme agro-écologie, il est possible que ce positionnement destiné à obtenir un «premium» sur les prix des produits vendus par la coopérative soit amené à évoluer. La coopérative prend déjà position en soulignant l'exemplarité de son action dans le cadre de la Loi sur l'avenir agricole. À ce propos, l'article cosigné par le directeur de Terrena dans la Tribune ${ }^{13}$ salue le projet de Loi mais le qualifie d' «étape non suffisante ». Enfin, la revue interne Horizons, à destination des adhérents de la coopérative affirme l'ambition de Terrena : être « le leader de l'agroécologie en Europe » (janvier 2013) ${ }^{14}$.

12. Voir http://agriculture.gouv.fr/politique-agricolecommune (consulté le 10 février 2015). Voir également annexe 2.

13. Laurent et Guillemin (2014): http://www. latribune.fr/opinions/tribunes/20140226trib 000817261/pour-une-strategie-nationale-agricolefrancaise.html

14. http://www.terrena.fr/uploads/pdf/97_ sommaire.pdf 


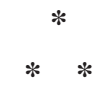

À travers l'approche discursive (Phillips et al., 2004), nous avons pu étudier l'institutionnalisation du concept d'agriculture écologiquement intensive en utilisant les articles de presse française. Ceux-ci ont formé un corpus exhaustif particulièrement intéressant: les sources sont autant légitimes que reconnues et tous les acteurs de l'institutionnalisation y sont représentés. L'analyse textuelle de cet ensemble de textes nous a permis i) d'identifier les discours associés à l'AEI, ii) d'analyser l'évolution chronologique de ces discours, iii) de mettre en relief les jeux d'acteurs et d'apporter des éléments de réponse à la question de l'institutionnalisation de l'AEI.

L'analyse longitudinale constitue un apport conséquent, car elle permet d'identifier que les discours sont caractéristiques de périodes. Dans un premier temps, l'AEI s'ancre dans le nouveau paradigme lié au développement durable, et prend son envol lors du Grenelle de l'Environnement (2007). Michel Griffon pose alors le concept et le développe particulièrement au sein de la coopérative agricole Terrena. C'est la phase d'objectivation de l'institutionnalisation. On observe alors les créations de l'association internationale pour une agriculture écologiquement intensive, et d'une Chaire AEI (2010-2012), pendant que le concept se diffuse et se partage au niveau local. Enfin, la dernière période suscite l'interrogation. Alors que le terme d'AEI semble avoir passé les différentes étapes de l'institutionnalisation, le processus de diffusion semble récupéré par l'acteur politique national qui met en avant l'agro-écologie. On observe un possible détournement d'opportunité qui est le fait de jeux d'acteurs. Contrairement à ce qui fut analysé pour les OGM (Brulé et Audebrand, 2009), il n'y a que peu de conflits ouverts autour de l'innovation que constitue l'AEI, mais de manière similaire, on observe différentes stratégies discursives concurrentes dont certaines s'inscrivent dans un objectif d'appropriation de l'innovation et certaines dans un cadre de remise en cause de ce nouveau cadre (voir Bonny, 2011; Goulet, 2012). L'acteur institutionnel s'imposant comme le porteur du concept bénéficie alors d'une appropriation qu'il peut valoriser vis-à-vis de ses parties prenantes. Le ministère de l'Agriculture, en institutionnalisant l'agroécologie, devient porteur d'un message et d'une politique, et, de plus, se retrouve soutenu par Terrena, du fait qu'il ait été acteur initiateur de l'AEI.

Une analyse prochaine du «parcours » de l'agro-écologie permettrait de confirmer ou d'infirmer ce détournement d'opportunité. Dans cet objectif, deux pistes de recherche semblent prometteuses. Dans un premier temps, il serait opportun d'utiliser la même méthodologie pour analyser les coupures de presse faisant référence à l'agro-écologie. Cela permettrait de mettre en relief le processus d'institutionnalisation mis ici en évidence pour l'AEI. Dans un second temps, analyser la réorganisation des filières de production permettrait d'éclairer le processus de changement institutionnel. En effet, Terrena ne semble pas avoir délaissé le terme AEI au profit de l'agro-écologie: les Terrenales en 2015 s'annoncent comme le « rendez-vous mondial de l'AEI», avec pour maîtres mots « l'innovation» et « l'agriculture de demain ».

Les auteurs remercient Sébastien Lantzi et Louis Sallenave, étudiants ingénieurs à AGROCAMPUS OUEST, CFR d'Angers, pour leur contribution aux analyses préparatoires à cet article, ainsi que Luc Audebrand pour sa relecture. 


\section{RÉFÉRENCES BIBLIOGRAPHIQUES}

Allaire G., Fuzeau V., Therond O. (2013). La diversité des cultures et l'écologisation de la Politique agricole commune. INRA Sciences sociales, Recherches en économie et sociologie rurales, $\mathrm{n}^{\circ} 1-2$, pp. 1-7.

Alvesson M., Karreman D. (2000). Varieties of discourse: On the study of organizations through discourse analysis. Human Relations, $\mathrm{n}^{\circ}$ 53, pp. 1125-1149.

Ben Slimane K. (2012). Retourner sa veste, toujours du bon côté : travail institutionnel discursif dans le déploiement de la télévision numérique terrestre en France. M@n@gement, vol. 15, n² 2, pp. 145-179.

Bonny S. (2011). L'agriculture écologiquement intensive: nature et défis. Cahiers Agriculture, vol. 20, n 6, pp. 451-462.

Bourdieu P. (1986). L'Illusion biographique. Actes de la recherche en sciences sociales, $\mathrm{n}^{\circ}$ 62-63, pp. 62-72.

Brulé E., Audebrand L. K. (2009). Changement institutionnel et stratégies discursives. Le cas des OGM en France (1996-2007). Revue française de gestion, $\mathrm{n}^{\circ}$ 194, pp. 83-104.

Capdevielle-Mougnibas V., Hermet-Landoi I., Rossi-Neves P. (2004). Devenir chercheur: rapport au savoir et engagement dans la recherche des doctorants en histoire et en mathématiques. Pratiques psychologiques, $n^{\circ} 10$, pp. 141-151.

Chahine S., Filatotchev I. (2008). The effects of information disclosure and board independence on IPO discount. Journal of Small Business Management, vol. 46, $\mathrm{n}^{\circ} 2$, pp. 219-241.

Chekkar R., Onnée S. (2006). Les discours managériaux dans le processus de communication financière: une analyse longitudinale du cas Saint-Gobain. Entreprises et Histoire, $\mathrm{n}^{\circ} 42$, pp. 46-63.

Conway G. (1998). The doubly green revolution. Ithaca, Cornell University Press.

De Raymond A. B., Goulet F. (dir.) (2014). Sociologie des grandes cultures. Au cœur du modèle industriel agricole, Paris, Quae.

DiMaggio P. J., Powell W. W. (1983). The Iron Cage Revisited: Institutional Isomorphism and Collective Rationality in Organizational Fields. American Sociological Review, vol. 48, n 2 , pp. 147-160.

Doise W. (1993). Debating social representations. Empirical approaches to social representations. Breakwell G. M., Canter D. V. (eds.), London, Blackwell, pp. 157-170.

Ernesto-Mendez V., Bacon C. M., Cohen R. (2013). Agroecology as a Trandisciplinary, Participatory, and Action-Oriented Approach. Agroecology and Sustainable Food Systems, vol. 37, n 1, pp. 3-18.

Geka M., Dargentas M. (2010). L'apport du logiciel Alceste à l'analyse des représentations sociales : l'exemple de deux études diachroniques. Cahiers internationaux de psychologie sociale, $\mathrm{n}^{\circ} 85$, pp. 111-135.

Ghali M., Daniel K., Colson F., Sorin S. (2004). L'agriculture écologiquement intensive. Une approche économique. Économie rurale, $\mathrm{n}^{\circ} 341$, pp. 83-99.

Goulet F. (2012). La notion d'intensification écologique et son succès auprès d'un certain monde agricole français : une radiographie critique. Courrier de l'environnement de l'INRA, n 62, pp. 19-29.

Grant D., Keenoy T., Oswick C. (eds.) (1998). Discourse and organization. London, Sage.

Greenwood R., Suddaby R., Hinings C. R. (2002). Theorizing change: the role of professional associations in the transformation of institutionalized rules. Academy of management Journal, vol. 45, $\mathrm{n}^{\circ}$ 271, pp. 58-80.

Griffon M. (2007). Pour des agricultures écologiquement intensives: des territoires à haute valeur environnementale et de nouvelles politiques agricoles. Groupe ESA, Angers, Les leçons inaugurales, décembre, $73 \mathrm{p}$.

Griffon M. (2013a). Qu'est-ce que l'agriculture écologiquement intensive? Versailles, Quae.

Griffon M. (2013b). Vers une septième révolution agricole. Projet, ${ }^{\circ}$ 332, pp. 11-19.

Hardy C, Phillips N. (1999). No joking matter: Discursive struggle in the Canadian 
refugee system. Organization Studies, $\mathrm{n}^{\circ} 20$, pp. 1-24.

Hardy C., Maguire S. (2008). Institutional Entrepreneurship. In R. Greenwood (ed.), The Sage Handbook of Organizational Institutionalism, Los Angeles, London, New Delhi, Singapore, Sage, pp. 198-217.

Helme-Guizon A., Gavard-Perret M.-L. (2004). L'analyse automatisée de données textuelles en marketing: comparaison de trois logiciels. Décisions Marketing, n 36, numéro spécial « Études qualitatives », pp. 75-90.

Illia L., Sonpar K., Bauer M. W. (2014). Applying Co-ocurrence Text Analysis with Alceste to Studies of Impression Management. British Journal of Management, $\mathrm{n}^{\circ} 25$, pp. 352-372.

Laurent C. Guillemin A. (2014). Pour une stratégie nationale agricole française. La Tribune, 26 février, disponible sur http://www.latribune.fr/opinions/tribunes/20140226trib 000817261/pour-une-strategie-nationaleagricole-francaise.html

Lawrence T. B. (1999). Institutional strategy. Journal of Management, $\mathrm{n}^{\circ}$ 25, pp. 161-187.

Lawrence T. B., Phillips N. (2004). From Moby Dick to Free Willy: Macro-Cultural Discourse and Institutional Entrepreneurship in Emerging Institutional Fields. Organization, September, ${ }^{\circ} 11$, pp. 689-711.

Lawrence T. B., Suddaby R. (2006). Institutions and Institutional Work. In S. Clegg, C. Hardy, W. R. Nord, T. Lawrence (eds.), Handbook of Organizational Studies, London, Sage, 2nd edition.

Le Foll S. (2013). Une vraie ambition pour l'agroécologie. Projet, n 332, pp. 20-23.

Maguire S., Hardy C. (2009). Discourse and Deinstitutionnalization: the Decline of DDT. Academy of Management Journal, vol. 52, $\mathrm{n}^{\circ} 1$, pp. $148-178$.

McInerney P. B. (2013). From Endogenization to Justification: Strategic Responses to Legitimacy Challenges in Contentious Organizational Fields. Organization Management Journal, vol. 10, n 4, pp. 240-253.

Merckaert J. (2013). Polyphonie pastorale. Projet, $\mathrm{n}^{\circ} 332$, pp. 2-3.

Michaud V. (2013) Business as pretext? Managing social-economic tensions on a social enterprise's websites.M@n@gement, vol. 16, n' 3, pp. 294-331.

Morgan G., Sturdy A. (2000). Beyond organizational change: Structure, discourse and power in U.K. financial services. London, Macmillan.

Moscovici S. (1976). La psychanalyse, son image et son public. Paris, PUF, $2^{e}$ éd.

Mumby D. K., Clair R. P. (1997). Organizational discourse. In T. A. van Dijk (ed.), Discourse as social interaction, London, Sage, pp. 181-205.

Munir K. A., Phillips N. (2005). The birth of the "Kodak moment": Institutional entrepreneurship and the adoption of new technologies. Organization Studies, ${ }^{\circ}$ 26, pp. 1665-1687.

Musson A. (2012). The build-up of local sustainable development politics: A case study of company leaders in France. Ecological Economics, ${ }^{\circ}{ }^{82}$, pp. 75-87.

Ostrom E. Basurto X.(2011). Crafting analytical tools to study institutional change. Journal of Institutional Economics, vol. 7, $\mathrm{n}^{\circ} 3$, pp. 317-343.

Pelletier P. (2010). Le néo-institutionnalisme sociologique en tant qu'ancrage théorique à la compréhension des arrangements institutionnels liés aux pratiques de gouvernance. Cahiers de recherche du Cergo, $\mathrm{n}^{\circ} 1$, http://www.cergo.enap.ca/CERGO/docs/ Cahiers_de_recherche/2010/Cahier_du_ CERGO_2010-01.pdf

Phillips N., Hardy C. (1997). Managing multiple identities: Discourse, legitimacy and resources in the U.K. refugee system. Organization, vol. 4, n 2 , pp. 159-185.

Phillips N., Hardy C. (2002). Understanding discourse analysis. Thousand Oaks, CA, Sage.

Phillips N., Malhotra N. (2008). Taking Social Construction Seriously: Extending the Discursive Approach in Institutional Theory. In R. Greenwood, C. Oliver, K. Sahlin-Andersson, R. Suddaby (eds.), The Sage Handbook of Organizational Institutionalism, Los Angeles, London, New Delhi, Singapore, Sage Publications, pp. 702-720.

Phillips N., Lawrence T. B., Hardy C. (2004). Discourse and Institutions. Academy 


\section{RECHERCHE}

Anne MUSSON, Damien ROUSSELIĖRE

of Management Review, vol. 29, $\mathrm{n}^{\circ} 4$, pp. 635-652.

Putnam L. L., Fairhurst G. (2001). Discourse analysis in organizations: Issues and concerns. In F. M. Jablin, L. L. Putnam (eds.), The new handbook of organizational communication: Advances in theory, research and methods, Newbury Park, CA, Sage, pp. 235-268.

Rao H., Monin P., Durand R. (2003). Institutional Change in Toque Ville: Nouvelle Cuisine as an Identity Movement in French Gastronomy. American Journal of Sociology, vol. 108, n ${ }^{\circ}$, pp. 795-843.

Reinert M. (1993). Les «mondes lexicaux » et leur «logique » à travers l'analyse statistique d'un corpus de récits de cauchemars. Langage et Société, $\mathrm{n}^{\circ}$ 66, pp. 5-39.

Reynaud E. (2001). Vers une meilleure compréhension des décisions stratégiques: l'apport de la méthode des scénarios. Finance, Contrôle, Stratégie, vol. $4, \mathrm{n}^{\circ} 2$, pp. 183-214.

Rousselière D., Vézina M. (2009) Constructing the legitimacy of financial cooperative in the cultural sector. A case study using textual analysis. International Review of Sociology, $\mathrm{n}^{\circ} 19$, pp. 241-261.

Scott W. R. (1987). The Adolescence of Institutional Theory. Administrative Science Quarterly, vol. 32, n 4, pp. 493-511.

Suddaby R., Greewood R. (2005). Rhetorical Strategies of Legitimacy. Administrative Science Quarterly, ${ }^{\circ}$ 50, pp. 35-67.

Suddaby R., Leca B. (Eds.), Institutional Work: Actors and Agency in Institutional Studies of Organizations. Cambridge University Press, pp. 236-261.

Vivien F.-D. (2005) Le développement soutenable. Paris, La Découverte.

Wezel A., Casagrande M., Celette F., Vian J.-F., Ferrer A., Peigné J. (2014) Agroecological practices for sustainable agriculture. Agronomy for Sustainable Development, $\mathrm{n}^{\circ} 34$, pp. 1-20.

Zilber T. B. (2007). Stories and the Discursive Dynamics of Institutional Entrepreneurship: The Case of Israeli High-tech after the Bubble. Organization Studies, $\mathrm{n}^{\circ} 28$, $1035 \mathrm{p}$. 
De quoi l'agriculture écologiquement intensive est-elle le nom ?

\section{ANNEXES}

\section{Annexe 1. Dendrogramme des classes constituant le corpus. Classification double}

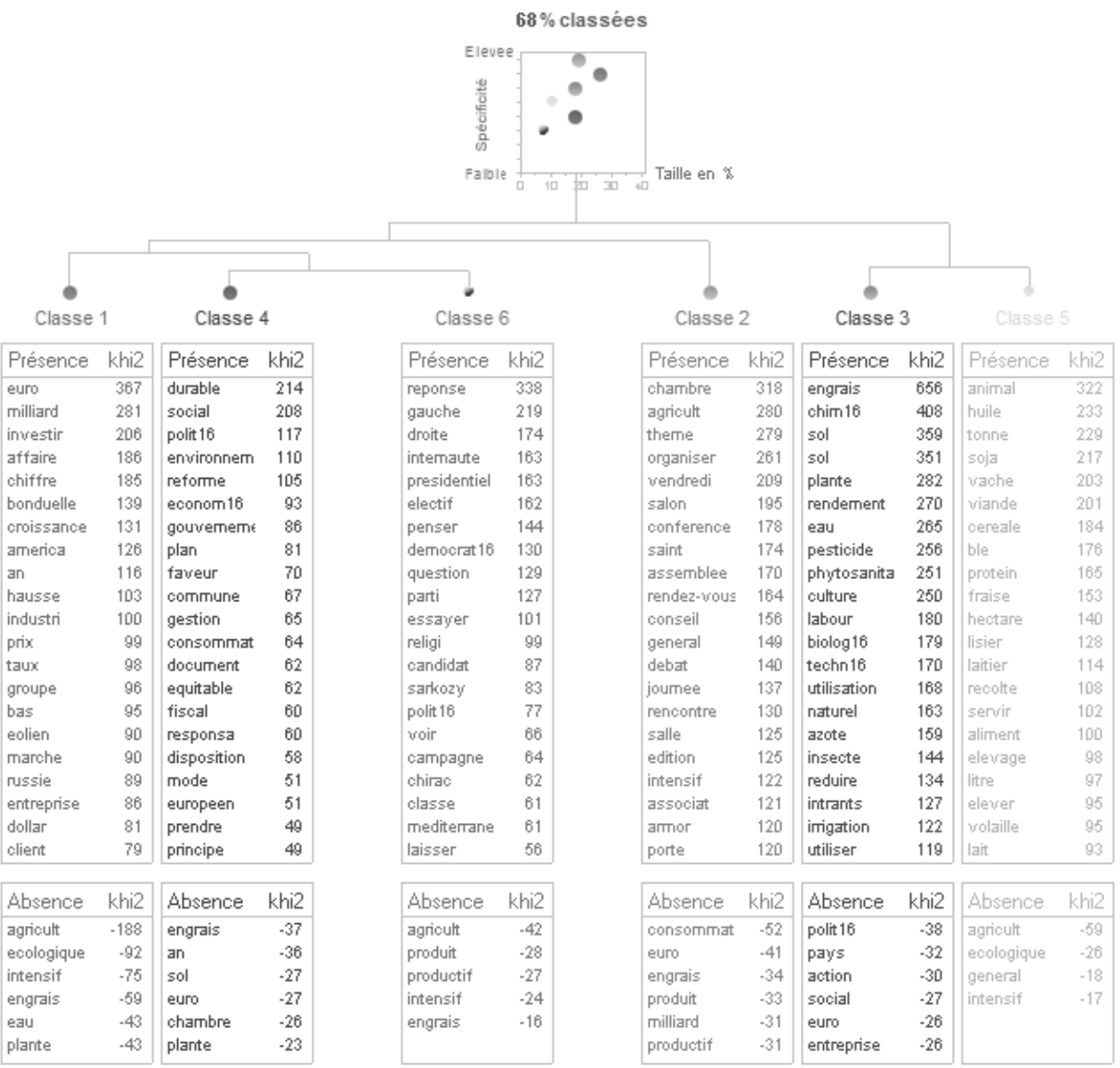

Source : les auteurs. 
RECHERCHE

Anne MUSSON, Damien ROUSSELIÈRE

\section{Annexe 2. La PAC et la Loi d'avenir agricole}

Copie d'écran du site internet du ministère français de l'Agriculture,

http://agriculture.gouv.fr/politique-agricole-commune (consulté le 10 février 2015).

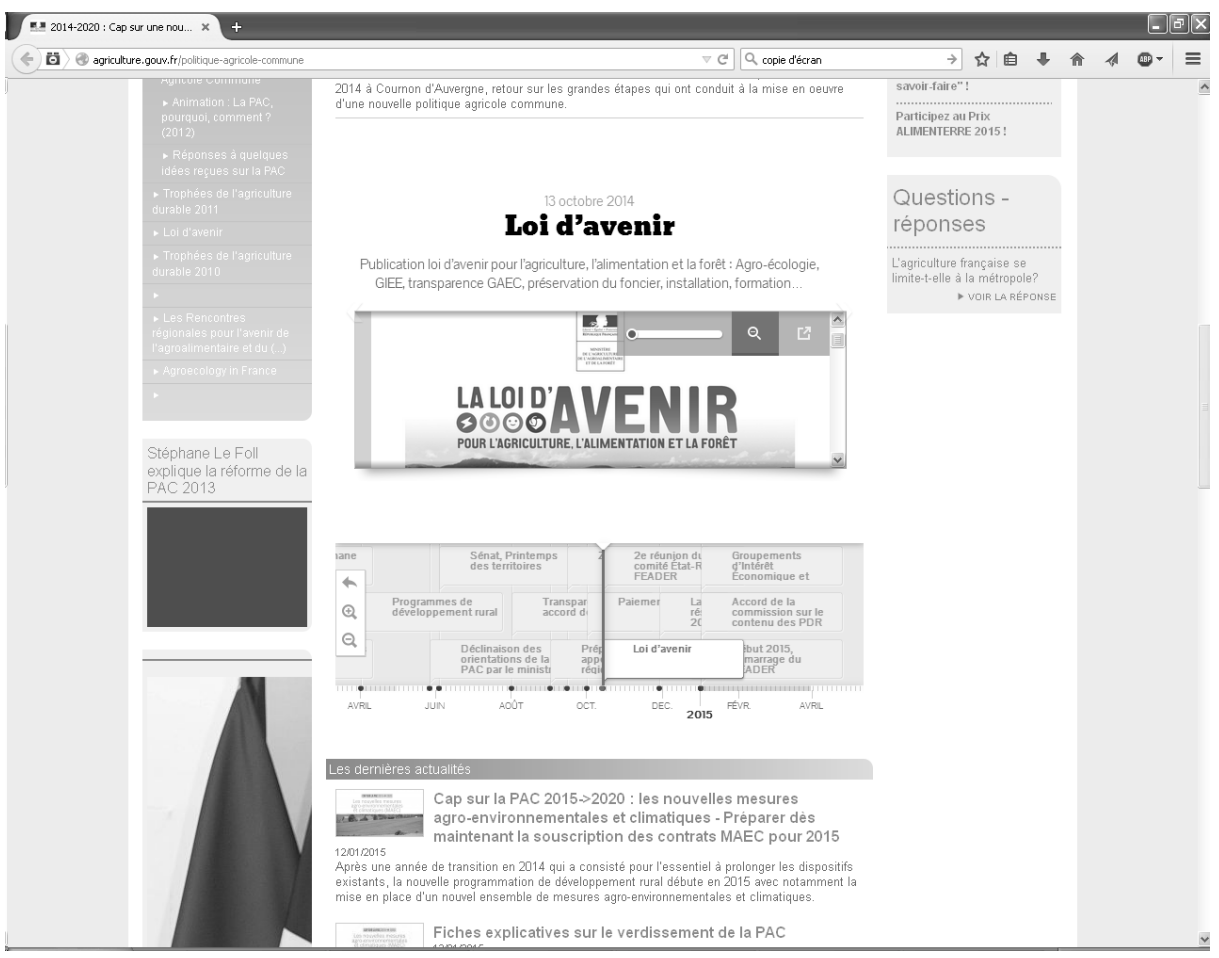

